\title{
ORIGIN OF THE TRANSIENT UNPULSED RADIO EMISSION FROM THE PSR B1259-63 BINARY SYSTEM
}

\author{
Lewis Ball ${ }^{1}$, Andrew Melatos ${ }^{2}$, Simon Johnston ${ }^{1} \&$ Olaf Skjæraasen ${ }^{1,3}$ \\ ${ }^{1}$ Research Centre for Theoretical Astrophysics, University of Sydney, NSW 2006, Australia; \\ ball@physics.usyd.edu.au \\ ${ }^{2}$ Miller Fellow, Department of Astronomy, 601 Campbell Hall, University of California, Berkeley CA 94720 USA \\ ${ }^{3}$ Institute of Theoretical Astrophysics, University of Oslo, P.O. Box 1029 Blindern, N-0315 Oslo, Norway
}

Astrophysical Journal Letters, in press

Original: 4 May 1998. Revised: 29 June 1998, 10 December 1998

Subject headings: binaries: eclipsing - circumstellar matter - stars: emission-line, Be radio continuum: stars - pulsars: individual: PSR B1259-63

\begin{abstract}
We discuss the interpretation of transient, unpulsed radio emission detected from the unique pulsar/Be-star binary system PSR B1259-63. Extensive monitoring of the 1994 and 1997 periastron passages has shown that the source flares over a 100-day interval around periastron, varying on time-scales as short as a day and peaking at $60 \mathrm{mJy}(\sim 100$ times the apastron flux density) at $1.4 \mathrm{GHz}$. Interpreting the emission as synchrotron radiation, we show that (i) the observed variations in flux density are too large to be caused by the shock interaction between the pulsar wind and an isotropic, radiatively driven, Be-star wind, and (ii) the radio emitting electrons do not originate from the pulsar wind. We argue instead that the radio electrons originate from the circumstellar disk of the Be star and are accelerated at two epochs, one before and one after periastron, when the pulsar passes through the disk. A simple model incorporating two epochs of impulsive acceleration followed by synchrotron cooling reproduces the essential features of the radio light curve and spectrum and is consistent with the system geometry inferred from pulsed radio data.
\end{abstract}




\section{Introduction}

The binary pulsar PSR B1259-63 is in a highly eccentric $(e \sim 0.87), 3.5$-yr orbit around a 10th magnitude B2e star, SS 2883, of radius $R_{*} \sim 6 \mathrm{R}_{\odot}$ (Johnston et al. 1992, 1994). The system is unique because PSR B1259-63 is the only radio pulsar orbiting a main-sequence companion with a circumstellar disk. Observations of the $\mathrm{H} \alpha$ emission line show that optically emitting material in the Be-star disk extends to at least $20 R_{*}$ (Johnston et al. 1994), and pulsar timing data suggest that the disk is steeply inclined to the plane of the binary orbit (Wex et al. 1998). The pulsar's orbital radius at periastron is $23 R_{*}$. Melatos, Johnston \& Melrose (1995) proposed a winddisk model of the system to explain orbital variations of the flux density, polarisation, rotation measure and dispersion measure of the pulsed emission near periastron (Johnston et al. 1996). In the model, the eclipse of the pulsed emission between $\mathcal{T}-20$ and $\mathcal{T}+16$ (where $\mathcal{T}$ denotes the epoch of periastron) is attributed to free-free absorption in the disk.

X-ray observations of PSR B1259-63 reveal significant (unpulsed) emission throughout the orbit, with the flux density at periastron $\sim 10$ times higher than at apastron (Hirayama et al. 1996). Modeling suggests that the $\mathrm{X}$-rays are synchrotron radiation from relativistic pulsar wind leptons accelerated at the shock between the pulsar wind and Be-star outflow (Tavani \& Arons 1997).

We discuss the origin of the transient, unpulsed radio emission detected from PSR B1259-63 from $\mathcal{T}-22$ until $\mathcal{T}+100$ (Johnston et al. 1999). The radio emission, like the $\mathrm{X}$-rays, is likely to be synchrotron radiation produced by the shock interaction between the pulsar wind and Be-star outflow. However, we argue that the interaction between the pulsar wind and an isotropic, radiatively driven, Be-star wind cannot explain the magnitude and rapidity of the variations in the radio light curve. Furthermore, we show that the radio emitting electrons, unlike the X-ray electrons, do not originate from the pulsar wind. We therefore suggest that the unpulsed radio emission results from the acceleration of electrons in the Be-star disk. The pulsar-disk encounter is likely to result in a strongly time-dependent, magnetized, hydrodynamical interaction forming a cometary pulsar wind nebula surrounded by a shock front where acceleration of particles is followed by a combination of adiabatic, synchrotron and inverse Compton cooling. However, the data so far obtained from this system are insufficient to constrain a model of the physics at this level of detail. Here we propose a simple model for the radio light curves which reproduces the main features of the data and identifies the essential physics.

\section{Observations}

Two periastron passages of PSR B1259-63 have been observed in detail, the first on 1994 Jan 9 and the second on 1997 May 29. Unpulsed radio emission was detected at frequencies between 0.84 and $8.4 \mathrm{GHz}$, as described by Johnston et al. (1999). Figure 4 of that paper shows the radio light curve at five frequencies during the 1997 periastron passage (the observed 1.4 GHz light curve is reproduced here in Figure 1b). Emission appeared suddenly at $\mathcal{T}-22$; prior to this the 3- $\sigma$ upper limit on the unpulsed emission is $\lesssim 0.5$ mJy at frequencies between 1.4 and $8.4 \mathrm{GHz}$. For the next few days, rapid variations were superimposed on an overall increase in the flux density $S$ at all frequencies, peaking at $S \sim 30 \mathrm{mJy}$ at $1.4 \mathrm{GHz}$ near $\mathcal{T}-8$. There followed a relatively slow decline before the flux density again began increasing around $\mathcal{T}+8$, peaking a second time near $\mathcal{T}+18$, with $S \sim 60 \mathrm{mJy}$ at $1.4 \mathrm{GHz}$. The flux density then declined steeply until around $\mathcal{T}+30$, when the rate of decay slowed to roughly that observed after the first peak near $\mathcal{T}-8$. This slower decline continued until the last observations on $\mathcal{T}+47$. The 1994 data are similar and showed detectable emission until $\mathcal{T}+100$.

At most times the radio frequency spectrum is well fitted by a power law $S \propto \nu^{-\alpha}$ of spectral index $\alpha \approx 0.5$, consistent with synchrotron radiation from relativistic electrons. Importantly, there is evidence in both the 1994 and 1997 data that the spectrum steepens after periastron (Figure 5 of Johnston et al. 1999). There is no compelling evidence for free-free absorption at any epoch between $\mathcal{T}-22$ and $\mathcal{T}+47$ (Johnston et al. 1999), even at periastron when the pulsed emission is totally eclipsed by the Be-star disk.

\section{Origin of the radiating electrons}

The relativistic pulsar wind is enclosed within a bubble as it ploughs through the material around the Be star. Momentum flux balance between the pulsar wind and Be-star outflow determines the size and shape of the bubble, and particles are shock accelerated at the boundary between the two flows (Melatos, Johnston \& Melrose 1995). 
The Be-star outflow consists of a radiation-driven polar wind and a more dense equatorial disk responsible for the bulk of the mass loss (Dougherty 1994). Both components are often characterised as simple power laws in radial distance $r_{\mathrm{s}}$, with number density and speed given by $n_{\mathrm{s}}=n_{0}\left(R_{*} / r_{\mathrm{s}}\right)^{\xi}$ and $v_{\mathrm{s}}=v_{0}\left(r_{\mathrm{s}} / R_{*}\right)^{\xi-2}$, where $\xi=2$ corresponds to a constant speed and $\xi=4$ corresponds to constant ram pressure. If the outflow is highly conducting so that the magnetic field is frozen into the plasma, then simple models imply $B_{\mathrm{s}} \propto r_{\mathrm{s}}^{1-\xi}$ for $2 \leq \xi \leq 3$. The polar wind is generally thought to reach a terminal speed $v_{\mathrm{w}} \sim 1000 \mathrm{~km} \mathrm{~s}^{-1}$ within a few stellar radii (e.g. Friend \& McGregor 1984); we therefore approximate it as a constant speed outflow with $\xi_{\mathrm{w}}=2$. In contrast, the disk density falls off rapidly with radius, as rotating material gradually accelerates outward; one typically has $\xi_{\mathrm{d}} \lesssim 4, v_{\mathrm{d} 0} \sim 5 \mathrm{~km} \mathrm{~s}^{-1}$ and a half opening angle of $\sigma_{\mathrm{d}} \sim 5-15^{\circ}$ (Waters, Coté \& Lamers 1987). The disk and wind number densities are related by $n_{\mathrm{d} 0} / n_{\mathrm{w} 0}=\left(v_{\mathrm{w}} / v_{\mathrm{d} 0}\right)\left(\dot{M}_{\mathrm{d}} / \dot{M}_{\mathrm{w}}\right)(1-$ $\left.\sin \sigma_{\mathrm{d}}\right) / \sin \sigma_{\mathrm{d}}$. For $\sigma_{\mathrm{d}}=10^{\circ}$ this implies a density ratio of $n_{\mathrm{d} 0} / n_{\mathrm{w} 0} \sim 10^{3}\left(\dot{M}_{\mathrm{d}} / \dot{M}_{\mathrm{w}}\right)$. Typical values of $\dot{M} \sim 2 \times 10^{-8} \mathrm{M}_{\odot} \mathrm{yr}^{-1}$ and $\dot{M}_{\mathrm{d}} / \dot{M}_{\mathrm{w}} \sim 10$ yield $n_{\mathrm{d} 0} \sim 4 \times 10^{18} \mathrm{~m}^{-3}$.

\subsection{Flux scaling for polar wind interaction}

If the spin-down luminosity of the pulsar, $\dot{E}_{\mathrm{p}}$, goes entirely into the relativistic wind then the ram pressure at a distance $r_{\mathrm{p}}$ from the pulsar is $\dot{E}_{\mathrm{p}} /\left(4 \pi c r_{\mathrm{p}}^{2}\right)$. The ram pressure of the Be-star polar wind is $m_{p} n_{\mathrm{s}}\left(r_{\mathrm{s}}\right) v_{\mathrm{s}}\left(r_{\mathrm{s}}\right)^{2}$. The contact discontinuity between the two flows has its apex at the point where the ram pressures balance, provided the magnetic pressures can be ignored. This point is denoted by $r_{\mathrm{p}}=D-r_{\mathrm{s}}=L$ where $D$ is the pulsar-Be star separation, $L / D=\sqrt{\eta} /(1+\sqrt{\eta})$ and $\eta=\dot{E}_{\mathrm{p}} /\left(\dot{M}_{\mathrm{w}} c v_{\mathrm{w}}\right)$ is the ratio of the momentum flux in the pulsar wind to that in the Be-star polar wind. The ratio $L / D$ is constant over the pulsar orbit, although $D$ and $L$ both vary.

Now suppose that the unpulsed radio flux is associated with the shock interaction between the pulsar wind and Be-star polar wind. Synchrotron theory implies that the flux density scales as

$$
S \propto n l^{3} B^{\alpha+1} \nu^{-\alpha}
$$

where $n$ is the number density of radiating electrons, $l$ is the source size and $B$ is the magnetic field. Assuming $l \propto L$ and that the other source parameters scale as their values at the contact surface apex, one obtains $l \propto D, n \propto D^{-2}$ and $B \propto D^{-1}$, implying $S \propto D^{-1 / 2}$ for $\alpha=0.5$ (irrespective of whether the radiating electrons originate from the pulsar wind or Bestar polar wind). Since $D$ varies by a factor of $\sim 15$ around the orbit, this argument implies that $S$ should be $\sim 4$ times higher at periastron than at apastron consistent with the sparse X-ray data, but far smaller than the $1.4 \mathrm{GHz}$ radio variation from $S \lesssim 0.5 \mathrm{mJy}$ at apastron to $S \sim 60 \mathrm{mJy}$ near periastron. Furthermore, in the interval near periastron, the above scaling yields $S(\mathcal{T}-27) \sim S(\mathcal{T}) / \sqrt{3} \sim 20$ mJy, yet at $\mathcal{T}-27$ there is no detectable unpulsed radio emission at any frequency. We conclude that the emission cannot be attributed solely to the interaction between the pulsar wind and Be-star polar wind.

\subsection{Disk or pulsar electrons? - Lorentz factor}

The pulsar orbit is eccentric and steeply inclined with respect to the Be-star disk, so the pulsar wind interacts strongly with the disk near periastron, potentially generating unpulsed radio emission. We now investigate whether the radiating electrons in this scenario originate from the pulsar wind or the disk.

The magnetic field in the emission region is the sum of the contribution from the pulsar, $B_{\mathrm{p}}(L)=$ $1.5 \times 10^{-3} R_{*} / L$ tesla (estimated from $P, \dot{P}$ and the assumption $B_{\mathrm{p}} \propto r_{\mathrm{p}}^{-1}$ in the pulsar wind), and the contribution from the Be star. The field in the Be-star polar wind satisfies $B_{\mathrm{S}}(D-L) \lesssim 10^{-2} R_{*} /(D-L)$ tesla (see Barker 1987 for an upper limit on surface fields of Be stars), while estimates of the field in the disk are so uncertain as to be of little value. For $L \lesssim D / 2$, one finds $B \sim 10^{-4} \mathrm{~T}$ at $D \sim 50 R_{*}$ when the radio peaks occur, and hence $B_{\mathrm{p}}(L) \sim B_{\mathrm{s}}(D-L)$. This value of $B$ is consistent with that obtained independently from synchrotron equipartition arguments, which yield $B \sim 10^{-4} \mathrm{~T}$ for $S(1 \mathrm{GHz}) \sim 50 \mathrm{mJy}$ and $\alpha=0.5$, assuming a source distance of $1.5 \mathrm{kpc}$, a spherical source of radius $25 R_{*}$.

The Lorentz factor required to produce synchrotron emission at $1 \mathrm{GHz}$ in such a field is $\gamma \sim 50\left(r_{\mathrm{p}} / 50 R_{*}\right)^{1 / 2}$ - five orders of magnitude lower than the likely Lorentz factor $\gamma \sim 10^{6}$ of unaccelerated leptons in the pulsar wind (Kennel \& Coroniti 1984; Arons \& Gallant 1994). We conclude that the pulsar-wind electrons are too energetic to be responsible for the unpulsed radio emission observed. On the other hand, 
non-relativistic electrons from the Be-star disk and/or polar wind could certainly be accelerated to the relatively modest energy required at $\operatorname{shock}(\mathrm{s})$ produced by the interaction between the pulsar wind and Bestar disk (e.g. Kirk 1994) as they are in binaries comprising early-type stars (Eichler \& Usov 1993).

\subsection{Disk or pulsar electrons? - Number density}

The number density of radiating electrons can be estimated straightforwardly for an assumed source size and magnetic field, since from synchrotron theory $n \propto S l^{-3} B^{-3 / 2}$. The source size $l$ is at least the standoff distance $L$, otherwise synchrotron selfabsorption would cause a detectable low-frequency turnover below $1 \mathrm{GHz}$ (Johnston et al. 1999). If the emission results from the interaction between the pulsar wind and Be-star disk, then the source is likely to be some kind of sheath around the contact discontinuity, extending downstream in a cometary tail. A spherical sheath with thickness $x L$, where $x$ is a numerical factor less than 1 , yields $n \sim x^{-1} 10^{10} \mathrm{~m}^{-3}$ for the parameters used above.

The number density of the pulsar wind can be estimated straightforwardly from the spindown luminosity $\dot{E}_{\mathrm{p}}=8.3 \times 10^{28} \mathrm{~W}$ and wind Lorentz factor $\gamma \sim 10^{6}$, yielding $n_{\mathrm{p}}\left(r_{\mathrm{p}}\right)=\dot{E}_{\mathrm{p}} / 4 \pi \gamma m_{e} c^{3} r_{\mathrm{p}}^{2}$ and hence $n_{\mathrm{p}}(L) \sim 2 \times 10^{4} \mathrm{~m}^{-3}$ for $L \sim 25 R_{*}$. This is at least 5 orders of magnitude smaller than the estimated number density required to produce the observed radio flux density. While the estimates clearly allow considerable latitude, and a shock may compress the wind by a factor of order 10, it is very difficult to argue away this discrepancy. We conclude that the pulsarwind electrons are too few in number to be responsible for the unpulsed radio emission observed. On the other hand, even if the number density of the Bestar disk falls off as steeply as $n_{\mathrm{d}} \propto r_{\mathrm{s}}^{-4}$, one finds $n_{\mathrm{d}}\left(25 R_{*}\right) \sim 10^{13} \mathrm{~m}^{-3}$, and acceleration of just $10^{-3}$ of the electrons in the disk can account for the observed emission.

\section{Model of the radio light curve and spectrum}

Johnston et al. (1999) and Wex et al. (1998) have argued that the Be-star disk is inclined with respect to the orbital plane in such a way that the pulsar passes through the disk twice in each orbit, once before periastron (crossing the disk around $\mathcal{T}-18$ at $D_{1} \approx 40 R_{*}$ ) and once afterwards $\left(\mathcal{T}+13\right.$ at $\left.D_{2} \approx 33 R_{*}\right)$. We postulate that the radiating electrons are created during time intervals $T_{1}$ and $T_{2}$ at the two disk crossings, when the shock interaction between the pulsar wind and the disk (and hence electron acceleration) is at its peak, and nowhere else. $T_{1}$ and $T_{2}$ are somewhat greater than the orbital crossing time of the disk ( $\sim 3$ days with $\sigma_{\mathrm{d}}=5^{\circ}$ according to Johnston et al. 1999) because the pulsar wind interacts appreciably with the disk surface for a few days before the pulsar enters and after it leaves.

The two resulting electron populations are assumed to evolve with time purely under the action of synchrotron losses. The loss times at the two crossings are given in terms of constants $b_{1,2}=$ $C\left(B_{1,2}^{2} / 2 \mu_{0}\right)$, where $B_{1,2}$ is the local magnetic field, $C=32 \pi r_{e}^{2} /\left(9 m_{e}^{2} c^{3}\right)$, and $r_{e}$ is the classical electron radius. The number densities per unit energy $N_{1,2}(\varepsilon, t)$ then obey

$$
\frac{\partial N_{1,2}(\varepsilon, t)}{\partial t}=b_{1,2} \frac{\partial}{\partial \varepsilon}\left[\varepsilon^{2} N_{1,2}(\varepsilon, t)\right]+Q_{1,2}(\varepsilon, t),
$$

where $Q_{1,2}(\varepsilon, t)$, the injection rate at each disk crossing, is a power law in energy during the times $T_{1}$ and $T_{2}$ and zero otherwise. Note that the evolution of the accelerated electrons depends only on conditions at the acceleration site; it is unrelated to the subsequent orbital position of the pulsar. Emission from the first disk crossing persists as the pulsar rounds the Be star (behind the disk) and is still present when the pulsar re-enters the disk after periastron, so the observed emission is the sum of that from the two electron populations.

The lack of evidence of a low-frequency spectral turnover implies that there is little absorption of the unpulsed radio emission (Johnston et al. 1999), even when the pulsar is at periastron and is obscured behind the dense Be-star disk. This strongly suggests that the radio-emitting electrons are left behind by the pulsar in a ram-pressure-confined bubble at the point where the pulsar crosses the disk, and that therefore their radio emission is not obscured by the bulk of the disk (Ball et al. 1998). We therefore ignore propagation effects on the radio emission.

The proposed model does not include the effects of adiabatic expansion in the evolution of $N_{1,2}(\varepsilon, t)$. Melatos, Johnston \& Melrose (1995) showed that when the pulsar is deep within the Be-star disk, the momentum flux in the pulsar wind is insufficient to disrupt the disk and so the pulsar wind is contained 
within a bubble. It follows that in a model which assumes that the bubble of radiating electrons is spherical, the source does not expand and so adiabatic losses do not occur. Electrons accelerated during each disk crossing are advected away from the shock apex in a cometary backflow (Melatos, Johnston \& Melrose 1995), but the observed rate of decline of the unpulsed radio emission indicates that the resulting adiabatic losses must occur on a timescale of a week or longer. In this Letter we show that a model involving only injection during the pulsar disk crossings and synchrotron losses is in qualitative agreement with the observations.

Inverse Compton losses may be important in this system (Tavani \& Arons 1997; Kirk, Ball \& Skjæraasen 1999). At the energies of the radio-emitting electrons these losses have the same form as synchrotron losses, and so the evolution of the electron spectrum will still be described by an equation of the form of (2). Outside of the disk the energy density of target photons from the Be star is comparable to the energy density in the magnetic field at the shock between the pulsar wind and the Be-star wind (Kirk, Ball \& Skjæraasen 1999), in which case the inverse Compton and synchrotron loss rates would be comparable. However, the density of target photons available to radio-emitting electrons originating in the disk is likely to be low because of screening by the disk (Tavani \& Arons 1997), and so inverse Compton losses are likely to be less important than synchrotron losses in the model for the radio emission proposed here.
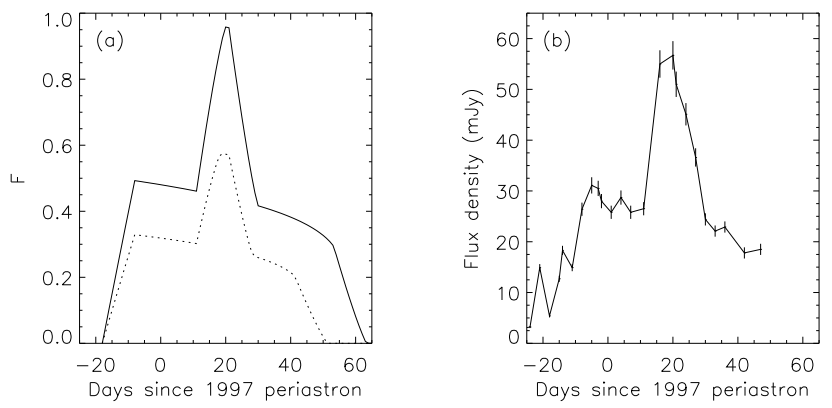

Fig. 1.- (a) Model flux density $F(\nu)$ in arbitrary units as a function of time (solid line), and at a frequency $\nu^{\prime}=1.44 \nu$ (dashed line). (b) Observed flux density from PSR B1259-63 at $1.4 \mathrm{GHz}$ (from Figure 4 of Johnston et al. 1999).

Assuming that the synchrotron emissivity can be approximated by a delta-function in frequency, the observed emission at a given frequency $\nu$ originates from electrons with characteristic energies $\varepsilon_{1}$ and $\varepsilon_{2}$ at the two acceleration sites. Since $\nu \propto B_{1,2} \varepsilon_{1,2}^{2}$ it follows that in general $\varepsilon_{1} \neq \varepsilon_{2}$. The total number density per unit energy of electrons emitting characteristically at $\nu$ is then $N_{1}\left(\varepsilon_{1}, t\right)+N_{2}\left(\varepsilon_{2}, t\right)$. When adiabatic losses (and therefore expansion) are neglected it follows from (1) that the that the emitted flux density is proportional to the number density of electrons radiating at $\nu$. The solid line in Figure 1a is the model flux density $F(\nu)$ obtained in this way, plotted in arbitrary units as a function of time. The curve is obtained by solving equation (2) for $T_{1}=T_{2}=10 \mathrm{~d}$, $1 /\left(b_{1} \varepsilon_{1}\right)=72 \mathrm{~d}, 1 /\left(b_{2} \varepsilon_{2}\right)=9 \mathrm{~d}$, and source functions of the form $Q_{1,2}(\varepsilon, t) \propto\left(\varepsilon / \varepsilon_{0}\right)^{-a}\left(\varepsilon_{\min } \leq \varepsilon \ll \varepsilon_{\max }\right.$, $a=2.2$ ). The dashed curve shows the model flux density for a frequency $\nu^{\prime}=1.44 \nu$. Since the pulsar is closer to the Be star during the post-periastron disk crossing than during the pre-periastron crossing, ram pressure balance implies $L_{2}<L_{1}$; however, the choice $L_{1} / L_{2}=4\left(=B_{2} / B_{1}\right)$ in Figure 1a is purely illustrative because we have no precise knowledge of the geometry of the pulsar-disk interaction. The synchrotron-loss times $1 /\left(b_{1,2} \varepsilon_{1,2}\right)$ are roughly the values expected for $B \sim 10^{-4} \mathrm{~T}$ and $\nu \sim 1 \mathrm{GHz}$; the injection intervals $T_{1,2}$ are roughly twice the pulsar disk crossing times; the relative magnitudes of $Q_{1}$ and $Q_{2}$ are chosen arbitrarily to be similar. The curves are approximately piecewise-linear, a characteristic of solutions to equation (2) for $a \approx 2$. The proposed model reproduces many of the essential features of the light curves in Figure 4 of Johnston et al. (1999), from which the $1.4 \mathrm{GHz}$ light curve is reproduced in Figure 1b. In particular, the flux density is higher after $\mathcal{T}+12$ because it is the sum of the contributions from two accelerated electron populations.

The following features of the light curves in Figures $1 \mathrm{a}$ and $1 \mathrm{~b}$ should be noted. (i) The first rise is associated with the pre-periastron disk crossing and lasts for a time $T_{1}$, because the synchrotron-loss time $1 /\left(b_{1} \varepsilon_{1}\right)$ is much greater than $T_{1}$. (ii) The gradual decay after $\mathcal{T}-8$, which continues until $\mathcal{T}+54$ (solid curve), occurs because the number of electrons per unit time cooling down to energy $\varepsilon_{1}$ from $\varepsilon>\varepsilon_{1}$ is slightly smaller than the number with energy $\varepsilon_{1}$ cooling to lower energies. (Exact balance occurs for $a=2$.) (iii) The second rise is associated with the post-periastron disk crossing and lasts for the synchrotron-loss time $1 /\left(b_{2} \varepsilon_{2}\right)$, because $T_{2}$ is larger than $1 /\left(b_{2} \varepsilon_{2}\right)$. (iv) The plateau at the principal max- 
imum lasts for a time $T_{2}-1 /\left(b_{2} \varepsilon_{2}\right)=1 \mathrm{~d}$, which is brief because the injection and loss times for the post-periastron disk crossing have been chosen to be almost equal. [cf. (ii)] (v) Following this brief plateau, $N_{2}\left(\varepsilon_{2}, t\right)$ decreases to zero over a time $1 /\left(b_{2} \varepsilon_{2}\right)$ equal to the rise time in (iii). Since $N_{2}\left(\varepsilon_{2}, t\right)$ decreases to zero relatively rapidly, its contribution drops below that of the more slowly decaying $N_{1}\left(\varepsilon_{1}, t\right)$ and the subsequent evolution is therefore an extension of (ii). Note that the pre-periastron rise time (i) is determined by injection (which lasts longer than the loss time), whereas the post-periastron rise time (iii) is determined by the loss time (which is shorter than the second injection episode). These rise times happen to be similar because we chose parameters such that $T_{1} \sim 1 /\left(b_{2} \varepsilon_{2}\right)$.

The changing separation of the solid and dashed curves in Figure 1a indicates that the effective spectral index $\bar{a}$ of the composite electron distribution is not constant. By crudely relating $\bar{a}$ to $\alpha\left(S \propto \nu^{-\alpha}\right)$ via $\alpha=(\bar{a}-1) / 2$, we predict that the spectrum: (i) has $\alpha \sim 0.7$ and does not change significantly from switch-on at $\mathcal{T}-18$ until the short plateau around $\mathcal{T}+20$; (ii) then steepens markedly with $\alpha$ increasing to $\sim 1.1$ before flattening again to $\sim 0.7$ at $\mathcal{T}+30$; (iii) steepens smoothly thereafter as the emission at higher frequencies decays more rapidly. The model behavior is broadly in accord with the data, which reveal a systematic steepening of the spectrum with the spectral index changing by $\sim 0.4$ between $\mathcal{T}+15$ and $\mathcal{T}+30$ (Figure 5 of Johnston et al. 1999), followed by a less pronounced flattening.

The model makes a clear, testable prediction. We see from Figure 1a that the slow decay after the first disk crossing (which continues well past the second disk crossing to the end of the observation period $\mathcal{T}+47$ ) ends sharply; the model flux density decreases to zero over a time comparable to the initial rise, $T_{1}=10 \mathrm{~d}$, instead of maintaining its very gradual downward slope all the way to zero. Although observations extending to $\mathcal{T}+100$ were made at $0.84 \mathrm{GHz}$ following the 1994 periastron, these single-frequency data are inconclusive as to the existence of such a feature. It will be possible to test this prediction during the next periastron passage; the frequency dependence of the onset of this rapid decline should also be observable.

The model makes no attempt to explain the shortest time scale variations in the radio emission. Particularly puzzling is the flat spectrum 'blip' which was observed at $\mathcal{T}-21$ in 1997 . We speculate that this feature is the result of the pulsar nebula splashing into the Be-star disk. Such effects are more likely to have observable consequences the first time the pulsar enters the disk because the system geometry is such that we have an unobscured view of the pre-periastron impact (see Figure 6 of Johnston et al. 1999), but not of the post-periastron impact.

\section{Conclusions}

We have argued that the transient, unpulsed radio emission from the binary system PSR B1259-63 is synchrotron emission associated with the shock interaction of the relativistic pulsar wind and the dense circumstellar disk of the Be star. The radiating electrons originate from the disk, not a spherically-symmetric pulsar wind with the parameters required to model the X-ray data.

We have presented a simple physical model for the evolution of the unpulsed radio emission, based on impulsive acceleration of electrons followed by synchrotron losses, which reproduces the salient features of the radio light curves and spectra observed during the 1994 and 1997 periastron passages. The model also makes a testable prediction of a rapid frequencydependent decay in the radio flux several weeks after the principal flux density peak, to be searched for during observations of the next periastron passage in October 2000.

O.S. would like to thank the Norwegian Research Council for support of this work through a postgraduate grant, and the RCfTA, University of Sydney for general support. A.M. gratefully acknowledges financial support from the Miller Institute for Basic Research in Science through a Miller Fellowship. 


\section{REFERENCES}

Ball, L., Melatos, A., Johnston, S., \& Skjæraasen, O., 1998, Memorie della Societa' Astronomica Italiana, in press

Dougherty, S. M., Waters, L. B. F. M., Burki, G., Coté, J., Cramer, N., van Kerkwijk, M. H., \& Taylor, A. R., 1994, A\&A, 290, 609

Eichler, D., \& Usov, V. V., 1993, ApJ, 402, 271

Friend, D. B., \& MacGregor, K. B., 1984, ApJ, 282, 591

Gallant, Y. A., \& Arons, J., 1994, ApJ, 435, 230

Hirayama, M., Nagase, F., Tavani, M., Kaspi, V. M., Kawai, N., \& Arons, J., 1996, PASJ, 48, 833

Johnston, S., Manchester, R. N., Lyne, A. G., Bailes, M., Kaspi, V. M., Qiao, G., \& D'Amico, N., 1992, ApJ, 387, L37

Johnston, S., Manchester, R. N., Lyne, A. G., Nicastro, L., \& Spyromilio, J., 1994, MNRAS, 268, 430

Johnston, S., Manchester, R. N., Lyne, A. G., D'Amico, N., Bailes, M., Gaensler, B. M., \& Nicastro, L., 1996, MNRAS, 279, 1026

Johnston, S., Manchester, R. N., McConnell, D., \& Campbell-Wilson, D., 1999, MNRAS, 302, 277

Kennel, C. F., \& Coroniti, F. V., 1984, ApJ, 283, 710

Kirk, J. G., 1994, in Kinetic Plasma Physics, Kirk, J. G., Melrose, D. B., \& Priest E. R., Springer-Verlag

Kirk, J. G., Ball, L., \& Skjæraasen, O., 1999, Astropart. Phys., in press

Melatos, A., Johnston, S., \& Melrose, D. B., 1995, MNRAS, 275, 381

Tavani, M., \& Arons, J., 1997, ApJ, 477, 439

Waters, L. B. F. M., Coté, J., \& Lamers, H. J. G. L. M., 1987, A\&A, 185, 206

Wex, N., Johnston, S., Manchester, R. N., Lyne, A. G., Stappers, B. W., \& Bailes, M., 1998, MNRAS, 298, 997

This 2-column preprint was prepared with the AAS LATEX macros v4.0. 